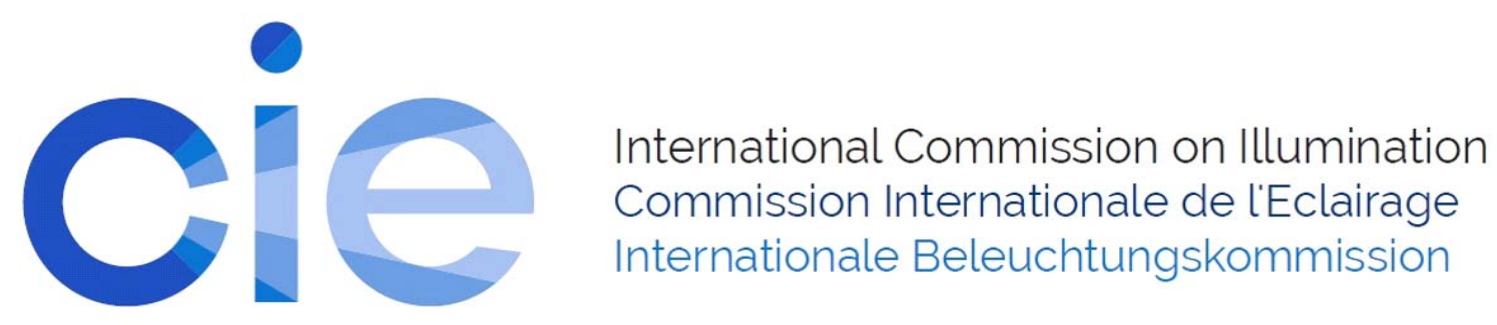

P0106

\title{
EVALUATION OF THE INFLUENCE OF AN INTEGRATING SPHERE INTERNAL STRUCTURE ON TOTAL LUMINOUS FLUX MEASUREMENT
}

Wataru shichi et al.

DOI 10.25039/x46.2019.PO106

from

CIE x046:2019

Proceedings

of the

29th CIE SESSION

Washington D.C., USA, June 14 - 22, 2019

(DOI 10.25039/x46.2019)

The paper has been presented at the 29th CIE Session, Washington D.C., USA, June 14-22, 2019. It has not been peer-reviewed by CIE.

\section{(C) CIE 2019}

All rights reserved. Unless otherwise specified, no part of this publication may be reproduced or utilized in any form or by any means, electronic or mechanical, including photocopying and microfilm, without permission in writing from CIE Central Bureau at the address below. Any mention of organizations or products does not imply endorsement by the CIE.

This paper is made available open access for individual use. However, in all other cases all rights are reserved unless explicit permission is sought from and given by the CIE.

CIE Central Bureau

Babenbergerstrasse 9

A-1010 Vienna

Austria

Tel.: +4317143187

e-mail: ciecb@cie.co.at

www.cie.co.at 


\title{
EVALUATION OF THE INFLUENCE OF AN INTEGRATING SPHERE INTERNAL STRUCTURE ON TOTAL LUMINOUS FLUX MEASUREMENT
}

\author{
Shichi, W. ${ }^{1}$, Toyota, T. ${ }^{1}$, Suzuki, T. ${ }^{1}$ and Ohkubo, K. ${ }^{2}$ \\ ${ }^{1}$ Industrial Research Institute of Shizuoka Prefecture, City of Shizuoka, JAPAN \\ ${ }^{2}$ Otsuka Electronics Co., Ltd., Koka, JAPAN \\ wataru2_shichi@pref.shizuoka.lg.jp
}

DOI 10.25039/x46.2019.PO106

\begin{abstract}
We examined the uniformity of the spatial response distribution function (SRDF) within an integrating sphere in which a baffle with a complex shape had been installed. The SRDF was measured by illuminating an inner surface of an integrating sphere with a spot light and also calculated by numerical simulation. Based on these SRDF results, we established a guiding principle for estimating the uncertainty of the total luminous flux measurement based on the difference in the light distribution between a test light source and a standard light source. Although differences of up to $0,4 \%$ were observed between the total luminous flux obtained in the numerical simulation and that obtained in the experiment, the basic overall tendency of the light distribution dependence was reproduced well in the simulation. This result suggests that it may be possible to apply numerical simulations to determine optimum baffle shapes.
\end{abstract}

Keywords: Integrating sphere, SRDF, total luminous flux, baffle shape, measurement uncertainty, numerical simulation

\section{Introduction}

The total luminous flux of an illumination light source is used as an objective value for measuring the total amount of light emitted by the relevant lighting, thereby allowing us to evaluate its luminous efficiency. A method using an integrating sphere is commonly used to measure the total luminous flux of a light source. In this method, the measured value of a test light source is compared with the measured value of a certified standard light source. If there is a difference in the light distribution between the test light source and the standard light source, a measurement error occurs due to the non-uniformity of the spatial response distribution function (SRDF) within the integrating sphere (Ohno, 1998; Ohno et al., 2001). In recent years, there have been many different light distributions for illumination sources, but the light distributions of standard light sources have been limited to just $4 \pi$ and $2 \pi$ light distribution sources. When measuring the total luminous flux of such illumination sources, the non-uniformity of the SRDF may affect the reliability of the measurement result. For this reason, it is important to identify the SRDF to evaluate the measurement uncertainty. One of the factors of SRDF non-uniformity is the internal structure of the integrating sphere. The SRDF is influenced by the internal structure of the integrating sphere, including the reflectivity of its inner surface, the acceptance angle sensitivity of the light detector, and the baffle. A baffle is a device that prevents the light detector from directly receiving the light emitted from the light source. According to several previous reports, baffles contribute significantly to the non-uniformity of the SRDF (Ohkubo et al., 2014; Wasapinyokul et al., 2017). The baffle installed in the integrating sphere that we used has a complex shape that is suitable for measuring both a spherical light source and a straight light source, such as a fluorescent light. As far as we know, the non-uniformity of the SRDF caused by a baffle with a complex shape and the uncertainty of the total luminous flux measurement caused by non-uniformity have not been discussed in any other reports. One purpose of this study is to analyse the influence of the baffle and other structures on the SRDF and the total luminous flux measurement by examining the SRDF of an integrating sphere with a complex baffle shape in both an experiment and a numerical simulation. Another purpose is to estimate and evaluate the error in the total luminous flux measurement from the SRDF when the light distribution of the test light source differs from that of the standard light source. In addition, this study also evaluates the usefulness of the numerical simulation by comparing the results of the experiment with those of the numerical simulation. 


\section{Methods}

\subsection{Experiments}

Figure 1 is a schematic diagram of the experiment. In this experiment, the SRDF of an integrating sphere with a diameter of $2 \mathrm{~m}$ (OptCom Co., LTD : LFM-080N) was measured by using a white LED spot light source. The colour temperature of the spot light source was 5300 $\mathrm{K}$. Located at the centre of the integrating sphere $(4 \pi$ placement $)$, the spot light source illuminated the wall surface with a spot circle that had a diameter of $120 \mathrm{~mm}$. The illuminating angle of the spot light source could be changed by 5 degree in both the horizontal and vertical directions. The vertical angle was measured by regarding the vertically downward direction as 0 degree. Figure 2 shows the light source unit (Ohkubo et al., 2019). Measurements could not be conducted for vertical angles of 150 degree or more due to the shape of the light source unit. The integrating sphere was equipped with a baffle for shielding direct light (Figure 2), an auxiliary bulb for measuring self-absorption, and a jig for attaching a measuring light source. The output signal was detected using a spectrometer from a detector port located at the same height as the centre of the sphere. We began taking measurements one hour after the spot light was turned on.

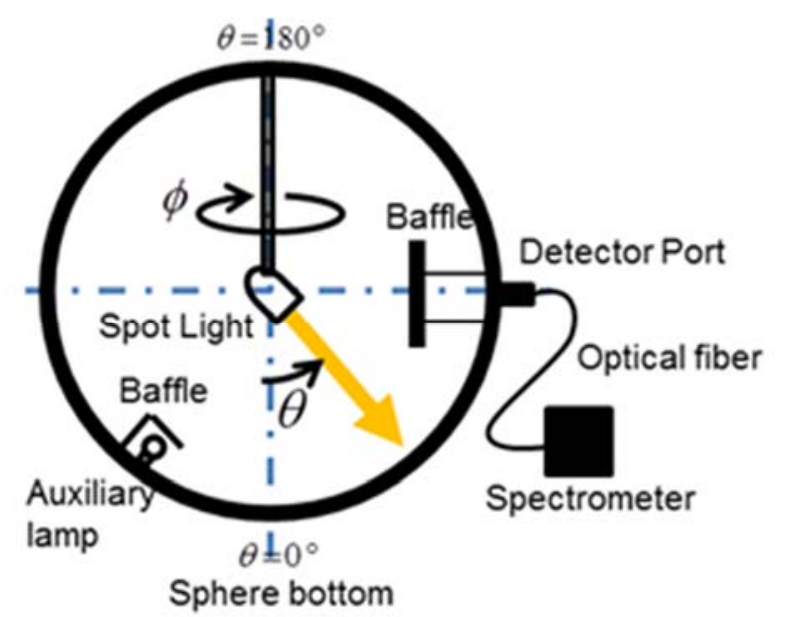

Figure 1 - Schematic diagram of the integrating sphere for SRDF measurement

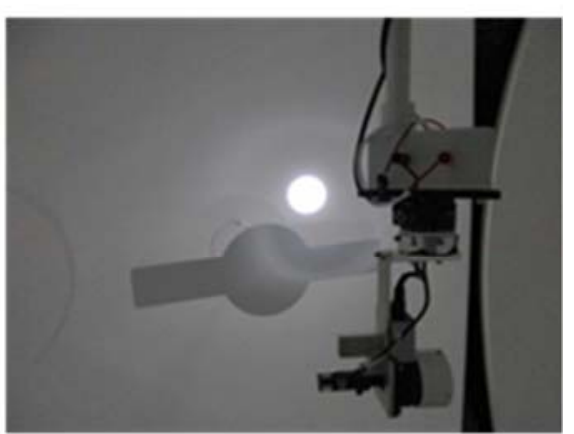

Figure 2 - SRDF light source unit and baffle

\subsection{Computer Simulation}

A numerical simulation was performed using the ray tracing software Lumicept (Integra Inc.). The integrating sphere model has the same shape and internal structure as the integrating sphere on which the experiment was conducted (Figure 3). The diffuse reflectance of the inner wall and internal structures of the integrating sphere was assumed to be $95 \%$ at all wavelengths, and all of the surfaces were assumed to be Lambertian. A spot light source for illuminating the inner surface of the integrating sphere with a spot circle of $120 \mathrm{~mm}$ in diameter was located at the centre of the integrating sphere. As was the case with the experiment, the light source illuminated the wall surface of the integrating sphere at 5 degree intervals in both the horizontal and vertical directions. The shape of the light source unit was not taken into account. The light receiving section was a $20 \mathrm{~mm} \times 20 \mathrm{~mm}$ rectangular region located in the same position as the integrating sphere that was used in the experiment, and its acceptance angle was $2 \pi$. The uncertainty associated with the reproducibility of the simulation result was $0,5 \%$. 

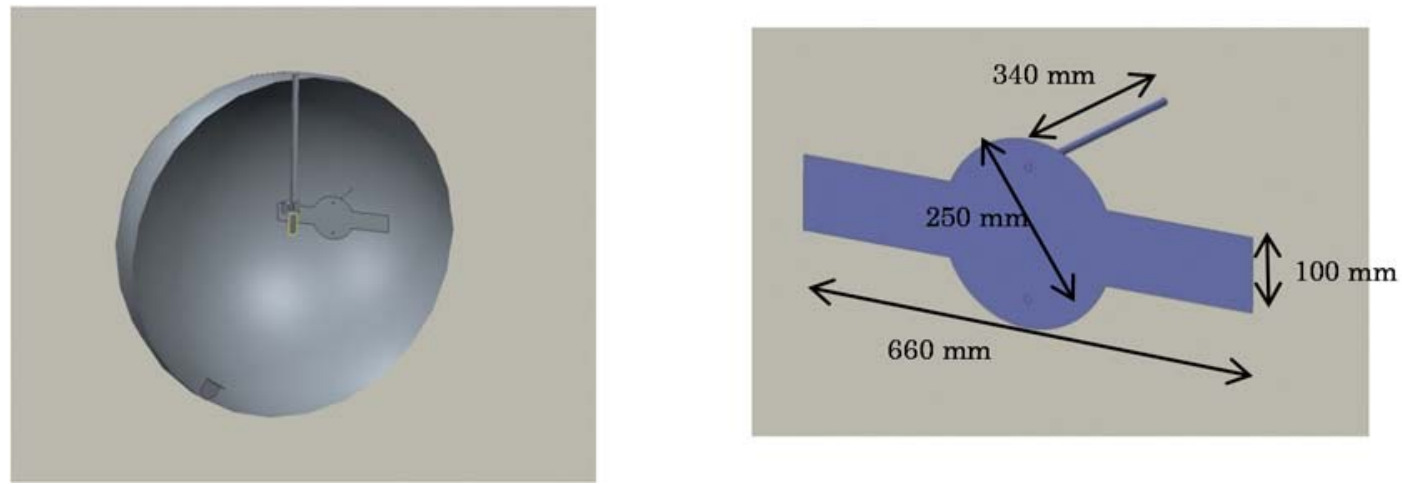

Figure 3 - Cross section of the integrating sphere model used for the numerical SRDF simulation and the baffle model

\section{Results and Discussion}

\subsection{SRDF of Integrating Sphere}

Figure 4 shows the SRDF obtained for the inside of the integrating sphere in the experiment, while Figure 5 shows the SRDF obtained for the inside of the integrating sphere in the numerical simulation. In both figures, the horizontal axis indicates the horizontal angle $(\phi)$, and the vertical axis indicates the vertical angle $(\theta)$. Both figures indicate values that have been normalized with respect to the average value of the obtained results. The light detecting section was located in the position corresponding to $\phi=225$ degree and $\theta=90$ degree, while the baffle was located $340 \mathrm{~mm}$ from the light detecting section in the direction toward the light source. Around this baffle, the SRDF value exhibited significant non-uniformity in excess of $5 \%$. In the region corresponding to between $\phi=0$ degree and $\phi=150$ degree, a non-uniformity of approximately $\pm 4 \%$ was observed due to the shadow of the baffle. At a point located at around $\theta=30$ degree in the region corresponding to between $\phi=60$ degree and $\phi=180$ degree, the influence of the supports for the baffle was observed. In the results of the experiment, non-uniformity due to the hemisphere edges that open and close the integrating sphere was observed at around $\phi=90$ degree and $\phi=270$ degree. This non-uniformity was not observed in the results of the numerical simulation because the hemisphere edges were not taken into account.
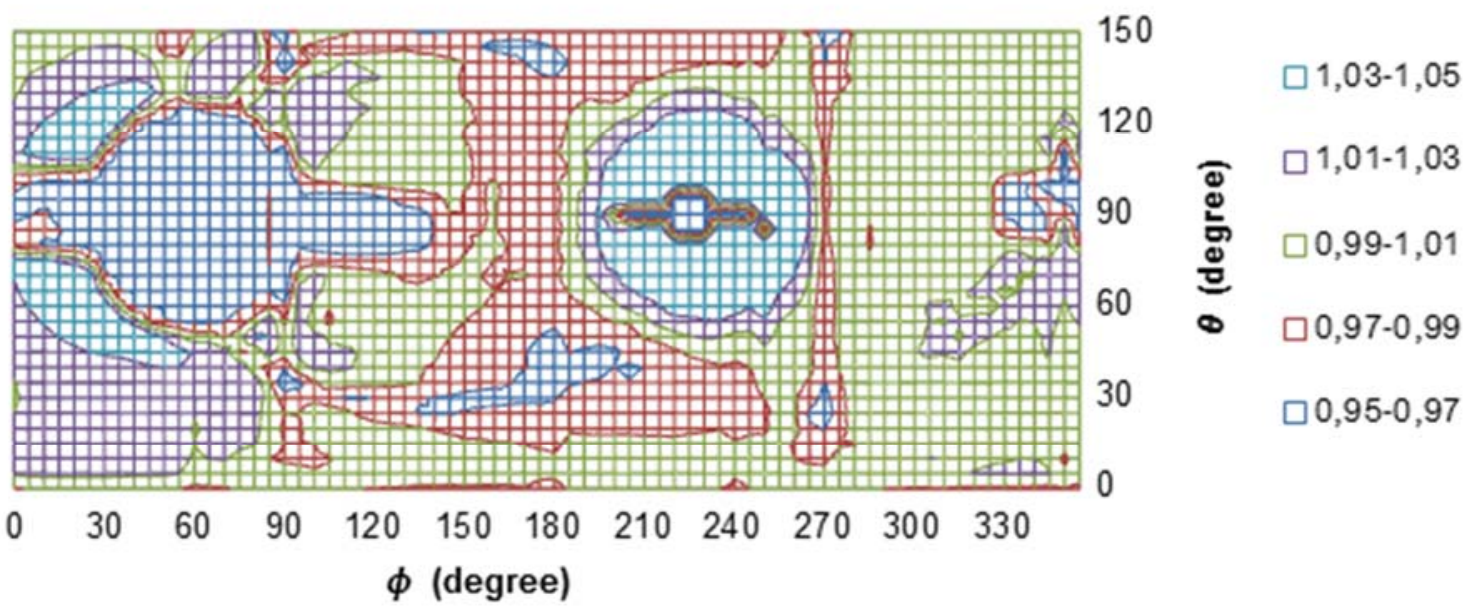

Figure 4 - Normalized SRDF obtained for the integrating sphere in the experiment 


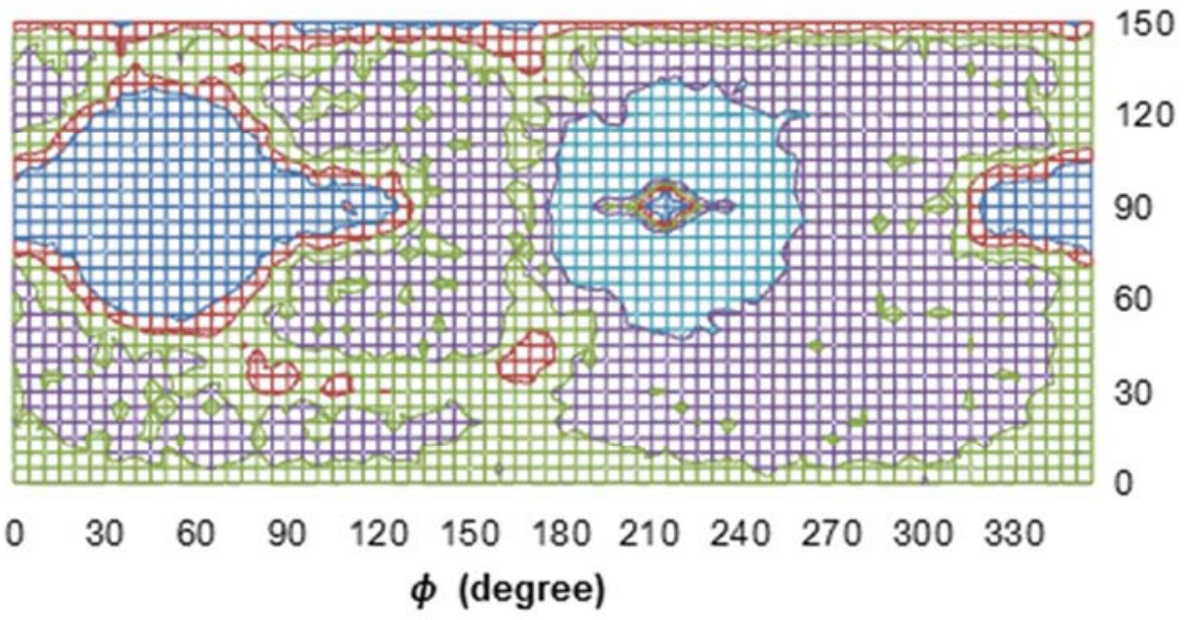

120

$\square 1,03-1,05$

잉 $\square 1,01-1,03$
힝 $\square 0,99-1,01$
릉 $\square 0,97-0,99$

$\square 0,95-0,97$

Figure 5 - Normalized SRDF obtained for the integrating sphere in the numerical simulation

\subsection{Total Luminous Flux}

To estimate the influence of the light distribution in the total luminous flux measurement, we calculated the total luminous flux for the SRDF obtained in the experiment and that obtained in the numerical simulation based on the assumption that a cone light source produced a conical light distribution with the cone angle $\alpha$, as illustrated in Figure 6 . The cone light source emitted light with its optical axis pointed in the vertically downward direction $(\theta=0)$ of the integrating sphere. The total luminous flux of the light source producing a conical light distribution with the cone angle $\alpha$ can be expressed by using the SRDF as follows.

$$
\Phi(\alpha)=\int_{\phi=0}^{2 \pi} \int_{\theta=0}^{\frac{\alpha}{2}} \mathrm{~K}(\phi, \theta) \mathrm{I}(\theta) \sin \theta \mathrm{d} \theta \mathrm{d} \phi
$$

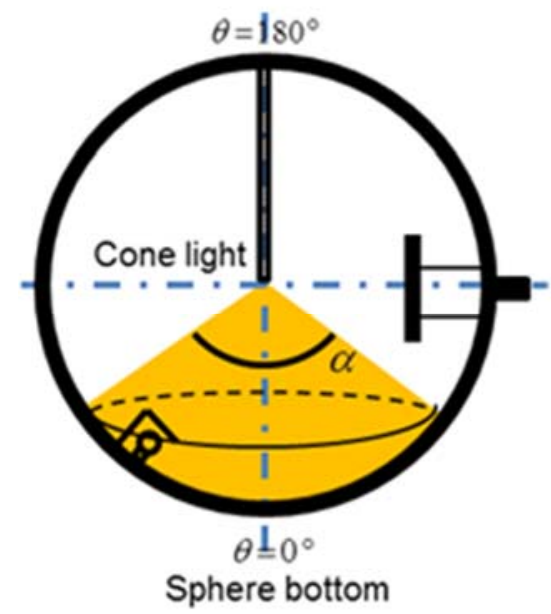

Figure 6 - Light distribution of a cone light source

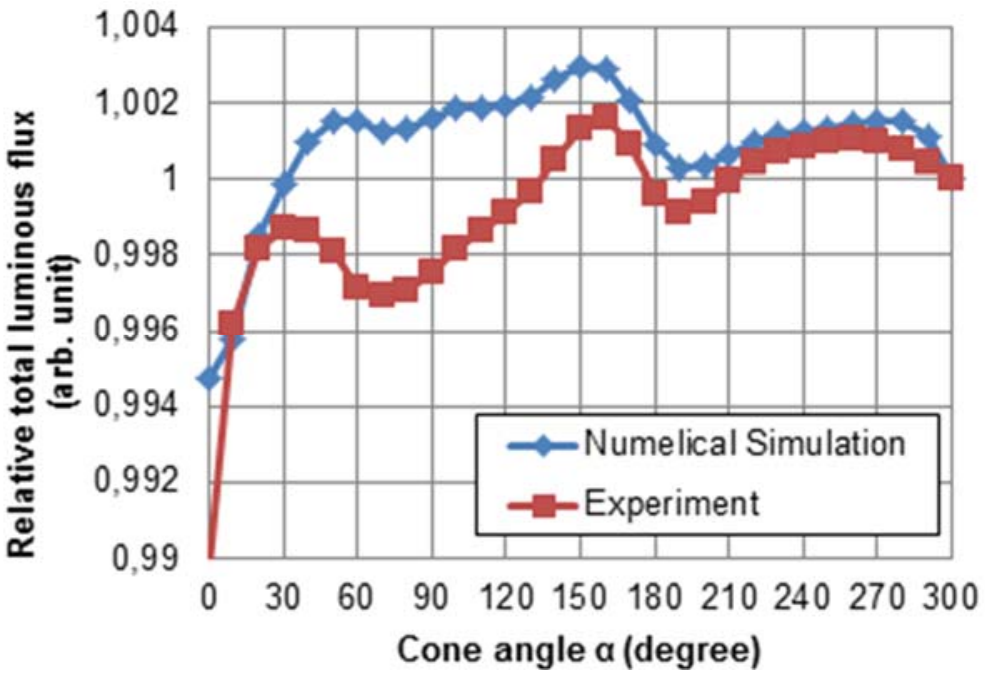

Figure 7 - Dependence of the total luminous flux on the cone angle $\alpha$ 
In this expression, $\mathrm{K}(\phi, \theta)$ is the SRDF of the integrating sphere and $\mathrm{I}(\theta)$ is the light distribution of the cone light source. Figure 7 shows the calculation results. In this figure, the points marked in red correspond to values calculated from Equation (1) by using the SRDF obtained in the experiment, while the points marked in blue correspond to values calculated from Equation (1) by using the SRDF obtained in the numerical simulation. The values indicated on the vertical axis have been normalized with respect to the value corresponding to a cone angle of $\alpha=300$ degree. Figure 7 shows that within the range of cone angles from 10 degree to 300 degree, the difference in the total luminous flux was $1 \%$ or less for both the experiment and the numerical simulation. Accordingly, if the cone angle of the measuring light source differs from that of the standard light source, errors of up to $1 \%$ may occur. The values obtained in the numerical simulation differed from those obtained in the experiment by up to $0,4 \%$ (around a cone angle of 70 degree). In the numerical simulation, simplified values were used for some of the parameters-such as the coating non-uniformity on the inner surface of the integrating sphere (Ohkubo et al., 2016) and the angle dependence of the detecting sensitivity (Ohkubo et al., 2012)-rather than the values obtained in the experiment. However, the cone angle dependence of the total luminous flux obtained in the numerical simulation reproduced the basic tendency of the measurement results. This result demonstrates that, compared with other factors, the internal structure (shape and arrangement) of the integrating sphere has a very large effect on the SRDF.

\section{Conclusions}

Our study results suggest that when a test lamp with a narrow light distribution, such as a spotlight, is measured by using a standard light source with a wide light distribution, such as a halogen lamp, the position and shape of the internal structure, especially the baffle, affect the measurement results of the total luminous flux. The simulation results also predict the measurement results with a reasonable degree of accuracy. Based on these results, we can estimate the uncertainty of this measurement. Furthermore, if it is necessary to reduce the measurement error of the total luminous flux, improvements to the non-uniformity of the SRDF can be considered by changing the shape and position of the baffle through computer simulation.

\section{References}

Ohno, Y.: "Detector-Based Luminous-Flux Calibration Using the Absolute Integrating-Sphere Method", Metrologia, 35, pp. 473-478 (1998).

Ohno, Y., and Daubch, O. R.: "Integrating Sphere Simulation on Spatial Nonuniformity Errors in Luminous Flux Measurement", Journal of the Illuminating Engineering Society, 30(1), pp. 105-115 (2001).

Ohkubo, K., and Mishima, M.: "Approximate Cosine Response Detector Head for Integrating Sphere Photometers", Journal of the Illuminating Engineering Institute of Japan, 96(11), pp. 761-766 (2012).

Ohkubo, K., and Osawa, Y.: "Effect to SRDF of Integrating Sphere in BRDF of Integrating Sphere Inside of Wall Reflective Material", Journal of the Illuminating Engineering Institute of Japan, 100(10), pp. 455-461 (2016).

Ohkubo, K., and Yamauchi, Y.: "Uncertainty of Photometry Caused by SRDF of Integrating Sphere", Journal of the Illuminating Engineering Institute of Japan, 103(4), pp. 142-147 (2019).

Wasapinyokul, K., Chuwongin, S., and Charoensook, A.: "Effects of Baffle Reflection and Shadow in an Integrating Sphere on the Total Luminous Measurement of a Linearly Shaped Lamp", Acta IMEKO, 6(4), pp. 105-112 (2017). 